\title{
Fractal properties of the IMF and the Earth's magnetotail field
}

\author{
K. Kabin and V. O. Papitashvili \\ Space Physics Research Laboratory, Department of Atmospheric, Oceanic, and Space Sciences, University of Michigan,
} 2455 Hayward St., Ann Arbor, MI 48109-2143, U.S.A.

(Received December 19, 1996; Revised September 26, 1997; Accepted September 29, 1997)

\begin{abstract}
The time series of the interplanetary and Earth's magnetotail magnetic fields are analyzed. The data subsets taken by the IMP 8 spacecraft outside and inside of the Earth's magnetosphere show clearly the fractal properties: their "box dimensions" are found to be close to 1.7 and 1.5 respectively. It is shown that data gaps have a little effect on the results. The magnetic field increment distributions are found to be non-Gaussian, but self-affine.
\end{abstract}

\section{Introduction}

Fractal properties of the interplanetary magnetic field (IMF) have been studied on many occasions. For example, Burlaga and Klein (1986) and Burlaga (1991) compared the fractal structure of the IMF near the ecliptic plane at $1 \mathrm{AU}$ and then at 6.0-8.5 AU. Ruzmaikin et al. (1993) utilized the fractal analysis to obtain asymptotics of the IMF spectra near 1.3 AU. Feynman and Ruzmaikin (1994) investigated the IMF spectra near the Earth's orbit and showed that the IMF distribution function is non-Gaussian. Therefore the power spectrum exponent is not the only parameter characterizing the MHD turbulence, although it is still a very important one. Burlaga (1991) and Marsch et al. (1996) showed existence of multifractal structures in the turbulent solar wind. In a recent paper, Ruzmaikin et al. (1995) studied turbulence in the fast solar wind data observed $b$ the Ulysses spacecraft at 3.9 AU deep in the Southern Heliosphere.

The purpose of this paper is to present some results of the comparison between fractal properties of the magnetic field measured by the IMP 8 spacecraft outside of the magnetopause and then in the Earth's magnetospheric tail. These results are obtained for 1.024-min averages of the threecomponent magnetic field measurements.

For the purposes of fractal analysis, the distribution of the increments in the time series is of greater importance than the distribution of the time series itself (Hastings and Sugihara, 1993); the latter can be obtained from the former via integration over the time increments and a shift to the mean value. We have investigated the magnetic field increments and found that their distribution function is nonGaussian but the important self-affinity property is still satisfied. This property ensures that the relationship

$$
\alpha=5-2 d
$$

holds for the IMF increments, as well as for the total field

Copy right ( $\odot$ The Society of Geomagnetism and Earth, Planetary and Space Sciences (SGEPSS); The Seismological Society of Japan; The Volcanological Society of Japan; The Geodetic Society of Japan; The Japanese Society for Planetary Sciences. magnitude (here $d$ is the "box dimension"; $\alpha$ is the magnetic field power spectrum exponent). Note that the self-affinity properties of the IMF increments have been guessed by Burlaga and Klein (1986) but seems never been tested directly. A seminal example where Eq. (1) does not hold can also be found in Tricot (1995, p. 169). Thus, the results of our study provide a firm mathematical basis for some of the earlier results obtained by different authors.

\section{Method}

To calculate the fractal dimension we used the apparent length of the curve, i.e., the data series. Our implementation of the technique follows the method developed by Higuchi (1988), rather than the earlier work by Burlaga and Klein (1986). If we have the observational time series of values $X\left(t_{i}\right)$ where time intervals are supposed to be equal, then the increments of $X$ can be defined as $\Delta\left(t_{j}-t_{i}\right)=X\left(t_{j}\right)-X\left(t_{i}\right), j$ $>i$. The non-normalized apparent length of the time series curve is defined as:

$$
L_{k}=\sum_{i}\left|\Delta\left(t_{i+k}-t_{i}\right)\right|
$$

If $X(t)$ is a fractal function, then the graph $\ln \left(L_{k}\right)$ versus $\ln (k)$ should be a straight line with a slope $1-d$ for small enough $\Delta_{k} t$. For large values of $k \Delta t$, the graph $\ln \left(L_{k}\right)$ versus $\ln (k)$ can obviously deviate from the straight line approximation because in this case it is derived for just a few data points chosen from the entire data set. In this range there is not enough statistical information to approximate the fractal properties of a given curve; therefore what we should be concerned with is the asymptotics of the slope for small $k \Delta t$.

The real-data time series may often have many gaps of the different length. We first tested our technique using a few data sets (with added gaps) of known fractal dimension, namely the Weierstrass-Mandelbrot function and the Brownian motion (Mandelbrot and van Ness, 1968). The test results provide us a firm confidence that the box dimensions obtained by the suggested technique agree well with the analytical results. This is one of the major advantages of fractal analysis over the conventional Fourier trans- 
form methods. Whenever we try to expand a discontinuous set of data in terms of smooth trigonometric functions, we should face Gibbs phenomenon (i.e., Lighthill, 1958), which can significantly distort the high frequency portion of the power spectrum. On the other hand, fractal analysis is free of the assumptions of data continuity; it can also access the asymptotics of the power spectrum directly. The disadvantage of the fractal analysis is that it cannot tell us much about the low frequencies, as a Fourier transform does, but this is not the subject of the present study.

Performing the real data analysis, we concluded that data gaps could be ignored as far as the fractal dimension does not change significantly over the length of the gap. In this way it becomes possible to divide the IMP 8 spacecraft data set in two separate time series for the corresponding IMF and magnetotail measurements; then we studied fractal properties of the data obtained for different space domains.

Despite the fact that, as mentioned above, many researchers have already studied the distribution functions of the IMF or other solar wind parameters, we think that the distribution of the IMF increments is also of great interest. For example, this distribution can be used to distinguish the IMF properties from those of the fractional Brownian motion. It turned out that these distributions are rather sensitive to the number of intervals used for evaluation. The maximum possible resolution is limited by the data accuracy and the time series length; in some occasions up to 200 intervals were used to obtain the distribution density with a maximum resolution.

\section{IMF 1-Minute Averages}

The IMP 8 spacecraft was launched by NASA in 1973 and continues to operate successfully to these days. The nearcircular 35 Earth's radii $\left(R_{\mathrm{E}}\right)$ 12-day orbit allows the spacecraft to spend about $40 \%$ of the time inside the magnetosphere measuring the Earth's magnetic field and plasma parameters in the far magnetospheric tail. During the rest of the time, the IMP 8 measures interplanetary parameters upstream of the magnetopause and bow shock. We analyzed one year of data from 1994 provided by the National Space Science Data Center: 1.024-min averages of the IMF three components (GSM $B_{x}, B_{y}$, and $B_{z}$ ) and total field magnitude $B$.

The spacecraft provides generally good data coverage during most of the time but significant data gaps frequently appear in the data stream due to a variety of reasons. Assuming that fractal properties of the IMF do not change significantly on the time scales up to a few days, we ignored all existing data gaps in the selected data set while applying Eq. (2). This also allows us to divide the data set in two orbital subsets. First, it is a part of the orbit with $X_{\mathrm{GSM}}>0$ which practically guarantees that the spacecraft was outside of the magnetopause; moreover, IMP 8 was even outside of the bow shock in most of the cases. Second, it is a part of the orbit with $X_{\mathrm{GSM}}<0$ and $\left(Y_{\mathrm{GSM}}{ }^{2}+Z_{\mathrm{GSM}^{2}}\right)^{1 / 2}<20 R_{\mathrm{E}}$, when the spacecraft was likely in the magnetotail. Such an approach has been utilized by a number of researchers, but mostly for the magnetic field power spectrum analysis (e.g., Russell, 1972a, b). However, according to Burlaga and Klein (1986), the fractal analysis is more economical and powerful method than the power spectrum analysis.
Figure 1 shows that the fractal dimensions of all three IMF components and the total field are approximately 1.7 (top panel). The fractal dimensions of the magnetotail field are $\sim 1.4$ for the total field and GSM $X$-component but $\sim 1.5$ for the GSM $Y$ and $Z$ components (lower panel). The differences between the dimensions of the $X$ component and total field and between the dimensions of $Y$ and $Z$ components inside the magnetotail are not large and might even be attributed to the imprecision of measurements and/or spacecraft magnetometer calibration. However, we should note that when our technique was tested for classical objects (i.e., the Weierstrass-Mandelbrot function and the Brownian motion, both taken with gaps), we obtained much better resolution (less than $3 \%$ for a set of $10^{5}$ points) than we seen in the experimental data. Of course, the real data have errors which are difficult to estimate and control and which can question the significance of the $7 \%$ difference in the results. However, we would like to draw attention to the fact that $X$ component of the magnetic field in the magnetotail is the largest, which suggests that there might be a physical explanation for the observed difference. Mathematics gives us a restriction that the box dimension of total field represented as $\sqrt{B_{x}^{2}+B_{y}^{2}+B_{z}^{2}}$ cannot be larger than the maximum box dimension of the components $B_{x}, B_{y}, B_{z}$ (for the bounded functions with a self-affine distribution of increments); similarly, any component of the magnetic field cannot have the box dimension larger than maximum of the box dimensions of two other components and the magnitude. We note that our dimensions are consistent with both parts of this restriction, which still however leaves the question weather the turbulence becomes anisotropic in the presence of a strong Earth's magnetic field unresolved.

We suggest that fractal properties of the magnetic field in the Earth's magnetotail are related to the turbulence in the
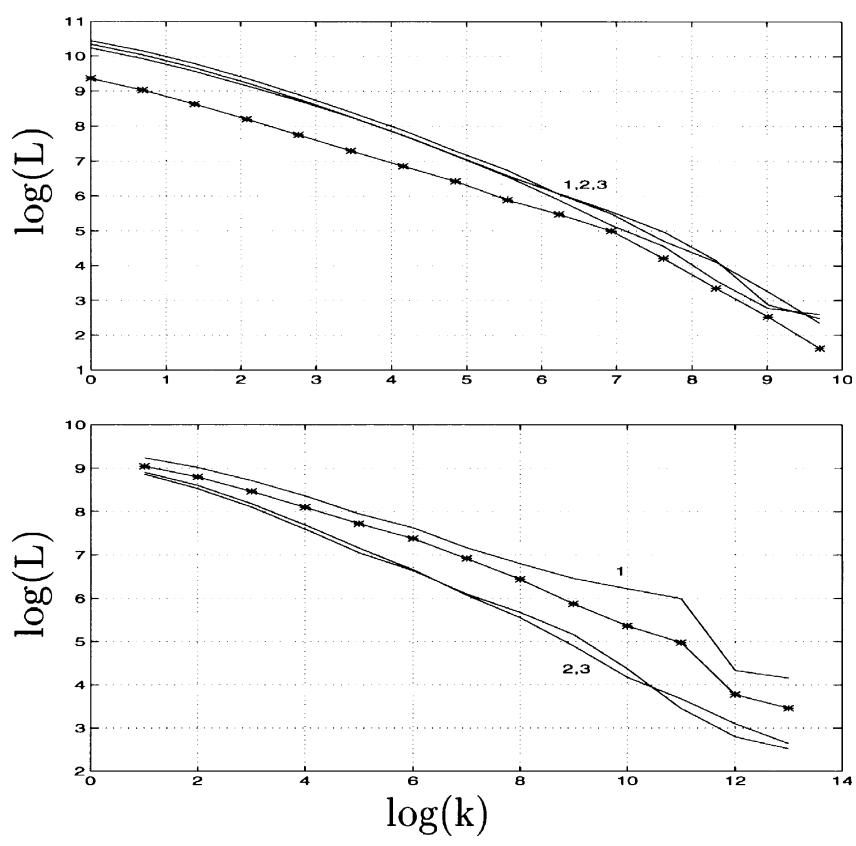

Fig. 1. Fractal dimensions of the magnitude (*) and three components (1 $-X ; 2-Y ; 3-Z$ ) of the IMF (top panel) and of the magnetotail field (bottom panel). 
IMF caused by the solar activity, rather than to the properties of the Earth's mostly dipolar field. The interaction of the solar wind with the Earth's magnetosphere is very complicated and not completely understood (see, i.e., Roberts, 1991). Obviously, this is a multidimensional nonlinear process, not a simple summation of two fields. The GSM $Y$ and $Z$ magnetic field components in the Earth's magnetospheric tail are much smaller in magnitude than the GSM $X$ component. Therefore, the former components may be affected by the solar wind turbulence to a larger degree and their expected fractal dimensions may be closer to those obtained for the interplanetary domain. This explanation suggests that there is an anisotropy in the near-Earth MHD turbulence.

An important reason for studying fractal dimensions of the IMF is the fact that they are related to the MHD turbulence power spectrum. Although for non-Gaussian process it is not the only characteristics of the turbulence (Feynman and Ruzmaikin, 1994) it is a primary parameter, which was the major object of theoretical and experimental research for decades. We note that the relationship (1) is correct only if a distribution of the field increments has the following property:

$$
F(\Delta x, \Delta t)=f\left(\frac{\Delta x}{\Delta t^{2-d}}\right)
$$

where $F(\Delta x, \Delta t)$ is the probability to find an increment in $x$ which is less than $\Delta x$ at time $t_{0}+\Delta t$, that is an integral of the probability density function (Mandelbrot and van Ness, 1968; Tricot, 1988; Hastings and Sugihara, 1993). The process can be Gaussian ( $f$ is an error function), as well as non-Gaussian. The key point here is that the function $F$ of two variables $\Delta x$ and $\Delta t$ can be reduced to a function $f$ of one variable $\Delta x / \Delta t^{2-d}$ (in this case $F$ is self-affine). We found that the magnetic field distributions under investigation are nonGaussian; this agrees with and might have been anticipated from the results presented by Feynman and Ruzmaikin (1994) for the IMF magnitudes. At the same time, these distributions still satisfy Eq. (3). Figure 2 (top panel) shows three density distribution functions of the total magnetic field magnitude in the solar wind with $\Delta t_{1}, \Delta t_{2}=2 \Delta t_{1}$, and $\Delta t_{3}$ $=4 \Delta t_{1}$ after appropriate transformations using the value $d$ evaluated before. In principle, one can imagine a method of estimation of the fractal dimension of a curve based completely on the distribution function of increments, but it is much more computationally complicated than the apparent length method. However, once $d$ is known, it is easy to check the self-affinity of the distribution functions. Therefore, we have used it just as an additional quality-control of our results. The vertical axis of the plot is $\ln (d f / d x)$ rather than $d f / d x$. The plots for the IMF components and for the magnetotail after the similarity transformation look almost identical to the upper panel of Fig. 2. It can be seen that property (3) is true to a very good approximation; the functions differ only in the distribution tails where the accuracy of calculations deteriorates anyway.

The important conclusion from this analysis is that the obtained distributions are very different from the Gaussian ones (the latter is plotted as a parabolic curve). We found
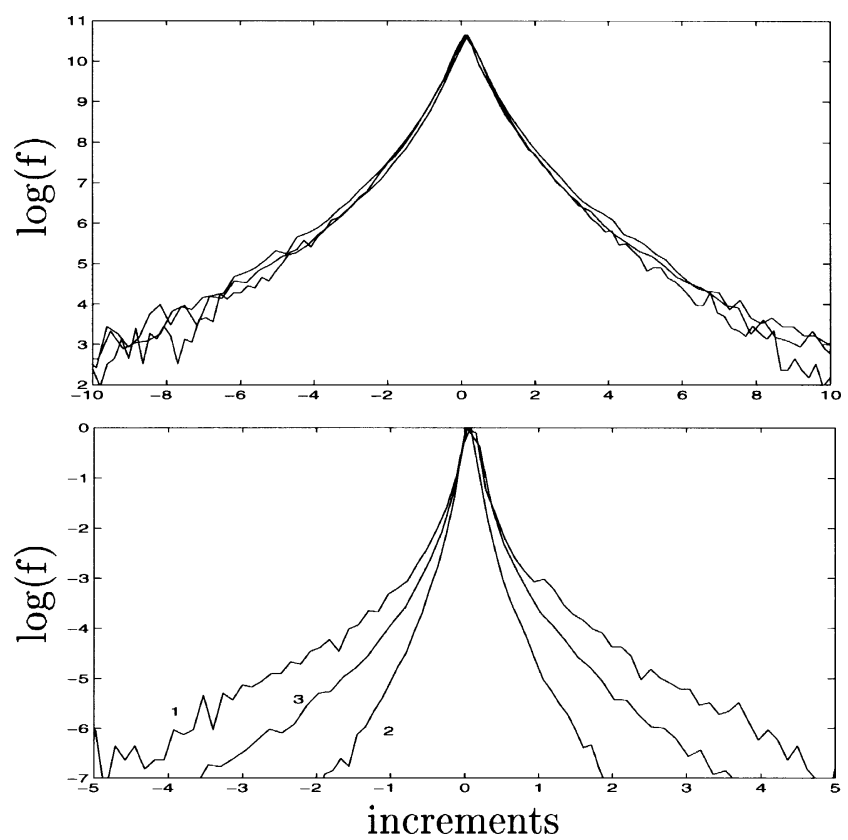

Fig. 2. Logarithm of the density distribution function: top - similarity transformation; bottom - (1) the magnetotail; (2) outside of the magnetosphere, and (3) all IMP 8 data combined for both domains.

that the relationship:

$$
\frac{d f(x)}{d x}=\frac{e^{-7|x|^{0.4}}}{1+x^{2}}
$$

provides a resonably good approximation to this kind of distribution functions. Therefore, we can conclude that fractal properties of the IMF are very different from those obtained for the fractional Brownian motion (e.g., Burlaga and Klein, 1986). In particular, it means that the method of a "midpoint displacement" (e.g., Crownover, 1995), often used for fractal interpolation of data, is not applicable for the IMF. IMF proprieties are more closely related to Lévy flights (see Shlesinger et al., 1995).

The distributions of the magnetic field increments inside the magnetospheric tail and outside of the bow shock are also shown on Fig. 2 (lower panel). This figure does not involve the similarity transformation (3) - it corresponds to a fixed value of $\Delta t$ (1.024 min). Curve 3 is given just for the comparison. Since the value $d$ is different in the magnetotail and outside of the bow sock, it is not self similar and would be significantly distorted if one would try to apply transformation (3) to the entire data set. As seen, the distribution of increments is much broader in the magnetotail where the magnitude of the magnetic field is larger, but it is still nonGaussian in its nature and has a similar form for all plotted cases.

\section{Conclusion Remarks}

The analysis performed in this study shows that the magnetic field measurements taken outside of the Earth's magnetosphere and then inside the magnetospheric tail are characterized by the non-Gaussian distributions of the field increments. These distributions have self-affine properties; 
therefore, the IMF power spectrum is related to its fractal dimension as suggested by Eq. (1). From Eq. (1) we can calculate the power spectrum exponent for our analysis: $\alpha=$ 1.6 outside of the bow shock; $\alpha=2.2$ inside the magnetotail. These numbers are in close agreement with results obtained by Russell (1972a, b): his values for the power exponents are 1.5-2 for the IMF and 2-2.5 for the magnetotail. We note that the "outer" exponent lays between two famous values: $\alpha=5 / 3$ which is the Kolmogorov's power low for isotropic homogeneous turbulence, and $\alpha=3 / 2$ which is the Iroshnikov-Kraichnan spectrum of pure Alfvenic turbulence.

Inside the magnetopause the influence of the Earth's magnetic field reduces the role of the solar wind turbulence, which is manifested in the reduction of the fractal dimension of the measured magnetic field. The power spectrum exponent increases, suggesting that the magnetic field becomes less turbulent. The obtained values of $\alpha$ show that the magnetic field outside of the magnetosphere is continuous but not continuously differentiable, while inside the magnetosphere the magnetic field has a continuous first derivative and a singular second derivative (e.g., Lighthill, 1959). According to Burlaga and Klein (1986), we can suppose that the rotational and tangential discontinuities inherent to the IMF are dissipated into weaker MHD singularities in the magnetotail. As seen on Fig. 1, the $Y$ and $Z$ components of the magnetic field inside the magnetotail are much greater affected by the solar wind turbulence than the total field and $X$ component magnitudes.

Acknowledgments. We would like to thank Andrew Nagy of SPRL for his interest to this work and fruitful discussions. The 1.024 min IMP 8 data are obtained from the NASA/National Space Science Data Center via the World Wide Web (http:// nssdc.gsfc.nasa.gov/space/imp-8.html). This work has been supported at the University of Michigan under funding from the Rackham Fellowship (KK) and the National Science Foundation grant ATM-9523329 (VOP).

\section{References}

Burlaga, L. F., Multifractal structure of the interplanetary magnetic field, Geophys. Res. Lett., 18, 69-72, 1991.

Burlaga, L. F. and L. W. Klein, Fractal structure of the interplanetary magnetic field, J. Geophys. Res., 91, 347-350, 1986.

Crownover, R. M., Introduction to Fractals and Chaos, 306 pp., Jones and Bartlett Publ., Boston, 1995.

Feynman, J. and A. A. Ruzmaikin, Distribution of the interplanetary magnetic field revisited, J. Geophys. Res., 99, 17,645-17,651, 1994.

Hastings, H. M. and G. Sugihara, Fractals: A User's Guide for the Natural Sciences, 235 pp., Oxford Univ. Press, Oxford, 1993.

Higuchi, T., Approach to an irregular time series on the basis of the fractal theory, Physica D, 31, 277-283, 1988.

Lighthill, M. J., Introduction to Fourier Analysis and Generalized Functions, 79 pp., Cambridge Univ. Press, Cambridge, 1958.

Mandelbrot, B. B. and J. W. van Ness, Fractional Brownian motions, fractional noises and applications, SIAM Review, 10, 422-437, 1968.

Marsch, E., C.-Y. Tu, and H. Rosenbauer, Multifractal scaling of the kinetic energy flux in solar wind turbulence, Ann. Geophys., 14, 259269, 1996.

Roberts, D. A., Is there a strange attractor in the magnetosphere?, $J$. Geophys. Res., 96, 16,031-16,046, 1991.

Russell, C. T., Comments on the measurement of power spectra of the interplanetary magnetic field, Solar Wind, NASA SP-308, 365-374, 1972a.

Russell, C. T., Noise in the geomagnetic tail, Planet. Space Sci., 20, 15411553, 1972b.

Ruzmaikin, A. A., I. P. Lyannaya, V. A. Stayshkin, and E. Yeroshenko, The spectrum of the interplanetary magnetic field near $1.3 \mathrm{AU}, J$. Geophys. Res., 98, 13,303-13,306, 1993.

Ruzmaikin, A. A., J. Jeynman, B. E. Goldstein, and E. J. Smith, Intermittent turbulence in solar wind from the south polar hole, J. Geophys. Res., 100, 3395-3403, 1995.

Shlesinger, M., G. Zaslavsky, and U. Frisch (eds.), Lévy Flights and Related Topics in Physics, 347 pp., Springer-Verlag, Berlin, 1995.

Tricot, C., Dimension fractale et spectre, J. de Chimie Phys. et de PhysicoChmie Biol., 85, 379-384, 1988.

Tricot, C., Curves and Fractal Dimension, 323 pp., Springer-Verlag, New York, 1995.

K. Kabin (e-mail: kabin@engin.umich.edu) and V. O. Papitashvili (e-mail: papita@umich.edu) 